\title{
PROGRAMA SEIS SIGMA EM PEQUENAS E MÉDIAS EMPRESAS: revisão e recomendações
}

\section{1- Carlos Henrique Mora Júnior}

Mestre em Administração pela Universidade Nove de Julho - UNINOVE, São Paulo/SP, Brasil. carlosmora@uninove.edu.br http://lattes.cnpq.br/3555208861939200

\section{2- Edmilson Lima*}

Doutor em Administração pela Ecole des Hautes Etudes Commerciales - HEC, Montreal, Canadá.

Professor do Programa de Pós-Graduação em Administração da Universidade Nove de Julho - UNINOVE, São Paulo/SP, Brasil.

edmilsonolima@gmail.com

http://lattes.cnpq.br/2700716884947412 


\title{
PROGRAMA SEIS SIGMA EM PEQUENAS E MÉDIAS EMPRESAS: REVISÃO E RECOMENDAÇÕES
}

\section{RESUMO}

Este artigo tem por objetivo fazer um levantamento do estado da arte a respeito do estudo de programas Seis Sigma em pequenas e médias empresas (PME) e sugerir alguns caminhos de pesquisa úteis para a expansão da fronteira de conhecimento sobre o tema. Os métodos empregados são baseados na revisão sistemática e organizada da literatura com o auxílio de diferentes classificações, tabelas e gráficos desenvolvidos especificamente para este estudo. Os resultados apontam a carência de estudos em geral sobre os programas Seis Sigma em contexto de PME, principalmente no que se refere a esses programas como estratégia competitiva nas PME, comparação do uso desses programas em PME e grandes empresas, estudos empíricos sobre seus fatores de sucesso em PME, modelos de implantação dos programas em PME e apreciação conceitual das implicações e potenciais do Seis Sigma em contexto de PME considerando-se as particularidades dessas organizações. Nas conclusões, sugere-se a realização de pesquisas sobre esses temas assim como de estudos que considerem a realidade do Seis Sigma em PME brasileiras.

\section{Palavras-Chave}

Programas seis sigma; pequenas e médias empresas (PME); estado da arte; revisão de literatura.

\section{SIX SIGMA PROGRAMS IN SMALL AND MEDIUM ENTERPRISES: LITERATURE REVIEW AND RECOMMENDATIONS}

\begin{abstract}
The objectives of this paper are to describe the state of art of Six Sigma studies in small and medium enterprises (SME) and to make some research suggestions to enlarge the frontiers of knowledge about the theme. The methods applied are based on the systematic and organized review of literature using a variety of classifications, tables and graphics specifically developed to this study. Results highlight research needs related to Six Sigma programs in general in the SME context, but mainly related to these topics: the use of those programs as competitive strategy in SME, comparisons of their use in SME and big enterprises, empirical researches about their success factors in SME, implementation models of those programs in SME and conceptual studies on the implications and potentialities of Six Sigma in SME considering the particularities of those organizations. The conclusions invite readers to do researches about those themes and studies that consider the reality of Six Sigma in Brazilian SME.
\end{abstract}

\section{Keywords}

Six sigma programs; small and medium enterprises (SME); state of art; literature review. 


\section{Introdução}

Seis Sigma é um programa de gestão baseado em técnicas estatísticas para reduzir a variabilidade e o desperdício dos processos em organizações (BANUELAS; ANTONY, 2002). Com base nesta redução, buscam-se a diminuição de custos e o aumento da qualidade na produção segundo a necessidade dos clientes. Do ponto de vista estatístico, Seis Sigma implica em 3,4 defeitos por milhão de eventos (produtos fabricados ou serviços realizados ou prestados), sendo sigma o termo usado para representar a variação em torno da média estatística observada no processo.

O maior estímulo das empresas para adotar o Seis Sigma está nos benefícios que este programa pode propiciar. Banuelas e Antony (2002) destacam que várias empresas têm relatado ganhos significativos com a implantação do Seis Sigma. Entre elas estão a General Electric - GE, que obteve mais de US\$ 2 bilhões em ganhos só no ano de 1999; a Motorola, que, após investir US\$ 170 milhões em treinamento, foi reconhecida com o prêmio Malcom Baldrige Award em 1988 e economizou US\$2,2 bilhões com a melhoria da qualidade em seus processos.

Casos de sucesso como estes são mais difíceis de serem identificados em pequenas e médias empresas (PME). Os desenvolvedores do Seis Sigma não focaram o método para este porte de organização (HARRY; CRAWFORD, 2004). Assim, pode-se perguntar: afinal, o Seis Sigma é aplicável às PME? Estas empresas não funcionam da mesma maneira que as grandes. Questões básicas de sobrevivência como a busca diária por capital de giro e demais recursos, assim como a entrega de produtos e serviços para clientes, consomem uma parcela extraordinária do tempo e da energia nas PME (MAIRANI, 2007). Como organizações frágeis, estas empresas raramente sobrevivem a falhas na gestão financeira, pois não haverá recursos suficientes para suportar a sua recuperação (WELSH; WHITE, 1981). Além disto, uma característica fundamental delas é a gestão centralizada do proprietário-dirigente.

Nos últimos anos, entretanto, a implantação do Seis Sigma em PME tem se tornado cada vez mais relevante. Muitas das grandes empresas têm exigido a implantação do programa Seis Sigma em sua base de fornecedores (pequenas indústrias) como uma condição para manter negócios com estas empresas. Tal condição passa a ser um desafio para essas PME, pois a implantação do Seis Sigma pode exigir altos investimentos, dedicação dos melhores recursos e treinamento de muitos empregados da empresa. As demais PME também estão disputando um mercado cada vez mais competitivo e o Seis Sigma Ihes é atrativo para a promoção da eficiência e da qualidade.

Neste ponto reside nosso interesse central: O que já foi estudado sobre este assunto? Como implantar o Seis Sigma em uma PME? Quais são os fatores críticos para sua implantação com sucesso? Existem modelos para isto? Quais são as adaptações necessárias? Quais são os resultados obtidos pelas PME que já implantaram o programa? A partir desses questionamentos, este trabalho tem dois objetivos: (1) revisar o estado da arte das pesquisas sobre o tema, organizando e sintetizando informações de revisão de literatura de acordo com um modelo de classificação, e (2) sugerir novos caminhos de pesquisas visando à expansão da fronteira do conhecimento sobre o tema. O presente estudo será útil especialmente para os iniciantes no tema, oferecendo a eles uma breve cartografia das variadas contribuições sobre o tema já feitas na literatura.

Este artigo foi estruturado da seguinte forma: na seção 2 é apresentado o método utilizado; na seção 3, é apresentada a revisão da literatura sobre Seis Sigma em PME; na seção 4, é apresentado o modelo de classificação dos 41 artigos revisados e uma tabela sintética dos artigos identificados e suas respectivas classificações; na seção 5, é feita uma análise geral dos estudos sobre o tema e, por fim, na seção 6 , desenvolvemos conclusões e recomendações úteis para novos estudos sobre o Seis Sigma em PME.

\section{Métodos}

O presente estudo é de natureza essencialmente bibliográfica. Para orientar este trabalho, foi utilizada uma estrutura metodológica proposta por Godinho Filho e Fernandes (2004), que é explicada pela figura 1.

Segundo esta estrutura, o passo inicial foi a realização de uma profunda revisão da literatura sobre Seis Sigma em PME. Foi realizada uma extensa busca em artigos de revistas acadêmicas, congressos da área da qualidade e gestão da produção, além de teses e dissertações. Foram utilizadas bases de dados reconhecidas tais como Proquest, Emerald e Scielo. Também foram consultados revistas e sites 
especializados no assunto, como: "Quality Digest Magazine", "Six Sigma Forum Magazine" e "www.isixsigma.com". Nestas fontes, foram encontrados 41 artigos sobre Seis Sigma em PME.

Figura 1 - Estrutura Metodológica

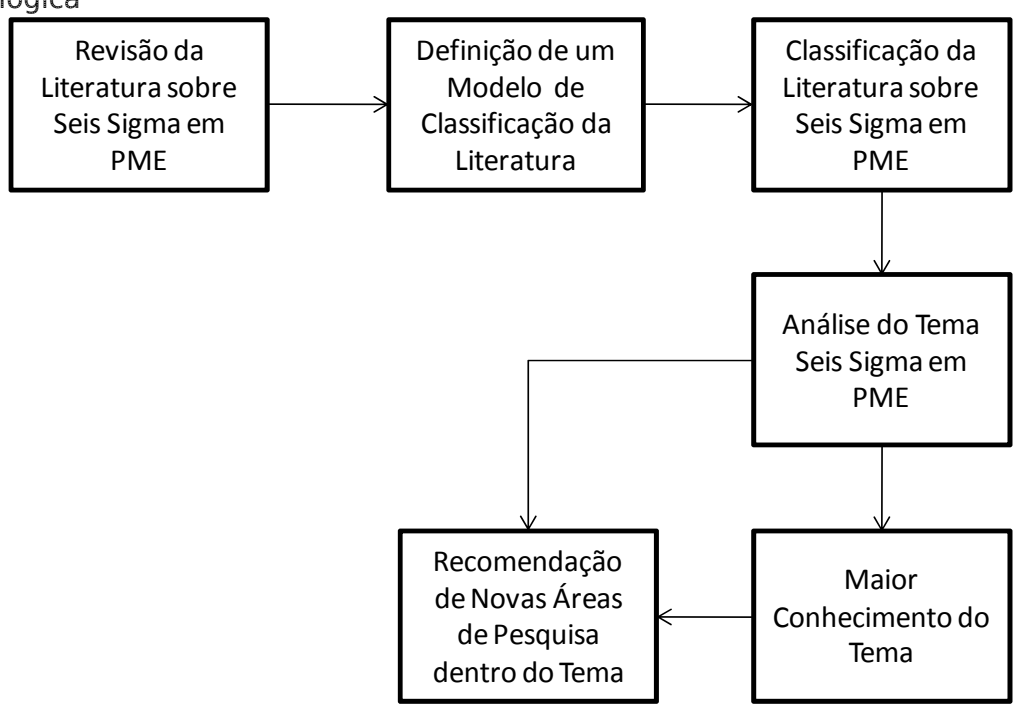

FONTE: Adaptado de Godinho Filho e Fernandes (2004).

Com base na revisão efetuada, o passo seguinte foi a definição de um modelo de classificação baseado em cinco parâmetros: tema, ano de publicação, origem da publicação, tipo do trabalho e método. A partir deste modelo, os 41 artigos revisados foram classificados e sintetizados em uma tabela resumo.

A revisão da literatura baseada na classificação dos trabalhos relevantes identificados propiciou uma análise detalhada do tema e o aprofundamento do conhecimento a seu respeito, o que permitiu a identificação de lacunas e oportunidades para a recomendação de novos temas de pesquisa sobre Seis Sigma em PME.

A seção seguinte trata das contribuições da literatura para se compreender o uso dos programas Seis Sigma nas PME.

\section{Seis Sigma em PME}

\subsection{O Gerenciamento da Qualidade e as Origens do Seis Sigma}

Não há consenso na literatura sobre o momento certo dos primeiros movimentos pela qualidade ou do gerenciamento da qualidade (DOW; SAMSON; FORD, 1999). Mesmo antes da revolução industrial, os artesãos já transmitiam de geração para geração suas experiências na manipulação de matéria-prima e produtos acabados (JURAN, 1989).

No século XVIII, a partir da revolução industrial, surgiram os primeiros instrumentos de medição e testes de laboratório e especificações escritas para matérias-primas, processos e produtos acabados. No fim do século XIX, foi observado um crescimento significativo da produtividade na indústria, muito influenciado pelos princípios da administração científica, que se fundamentava na separação entre o planejamento e a execução do trabalho. Este crescimento veio acompanhado de problemas de qualidade dos produtos e processos. Para contornar estes problemas, os gestores da época criaram departamentos para inspeção dos produtos após a produção (JURAN, 1989).

No início do século $X X$, o crescimento da oferta e da demanda por produtos e o aumento da complexidade dos processos produtivos implicaram no surgimento da engenharia da qualidade e engenharia da confiabilidade, que se baseavam no controle estatístico e na redução do número de defeitos. Segundo Juran (1989), este movimento ganhou força com a grande demanda por equipamentos militares de alta qualidade durante a segunda guerra mundial. Nesta época (década de 1940), surgiu a série ISO 9000 com o desenvolvimento de requerimentos técnicos e especificações para equipamentos militares (BASU; WRIGHT, 2003). 
Passada a segunda guerra, com o movimento para a reconstrução do Japão, apoiado por consultores americanos e europeus, entre eles Deming e Juran, surgiu a gestão da qualidade total (TQM). Ela envolve toda a organização com o objetivo de implementar melhorias na qualidade orientadas para o cliente (BASU; WRIGHT, 2003; DEMING 2000).

Nesta mesma época, surgiu no Japão, mais especificamente na Toyota, o sistema Toyota de produção, também conhecido por manufatura enxuta. Segundo Womack et al. (1992), Eiji Toyoda e Taiichi Ohno perceberam que a produção em massa não funcionaria no Japão e, então, desenvolveram uma nova abordagem para a produção que tinha como alvo principal a eliminação de desperdícios.

Na década de 1970, pressionada pela entrada das empresas japonesas, a empresa americana Motorola desenvolveu um plano de renovação que tinha como objetivo aumentar a qualidade de seus produtos em dez vezes. Visando definir modos de mensuração para obter tal melhoria, a companhia, na época, decidiu utilizar o sigma (desvio padrão) (BARNEY; MCCARTY, 2002). Daí surgiu o termo Seis Sigma, que, do ponto de vista estatístico, implica em 3,4 defeitos por milhão de produtos fabricados ou serviços realizados. Com o forte envolvimento do então presidente da Motorola, Bob Galvin, esta iniciativa gerou frutos e se consolidou.

A partir de então, o programa Seis Sigma foi adotado por várias outras empresas como Texas Instruments (1988), IBM (1990), Asea Brown Boveri (1993), Allied Signal e Kodak (1994), mas alcançou seu auge de popularidade com os significativos resultados reportados pela General Electric no final da década de 1990, período em que era presidida por Jack Welch (SENAPATI, 2004). No Brasil, o Seis Sigma foi introduzido em 1997, a partir de sua utilização pelo grupo Brasmotor (WERKEMA, 2002).

\subsection{Modelos de Implantação do Seis Sigma em PME}

Inicialmente, a adoção do Seis Sigma foi observada primordialmente em grandes organizações com um alto volume de processos de alta complexidade. Contudo, defende-se que não há restrição para a aplicação deste programa em PME (WESSEL; BURCHER, 2004) com a obtenção de bons resultados (THOMAS, 2006 ).

Boa parte dos modelos propostos na literatura para implantação do Seis Sigma em PME leva em consideração o contexto destas organizações, principalmente em relação às suas restrições financeiras, indicando formas de implantação com investimento reduzido (SCHWINN, 2003; BURTON, 2004; PINHO, 2005). Assim, um dos modos para a sua adoção é a implantação com um ritmo que a empresa possa suportar dentro de suas capacidades (BURTON, 2004), com os novos investimentos sendo realizados somente após o retorno dos primeiros (SCHWINN, 2003). Para Gross (2001), a PME em relação forte com uma grande organização que usa o Seis Sigma deve buscar apoio desta última para se inserir neste programa com custos reduzidos de implantação.

Em relação ao modelo de implantação em si, cada autor partiu de uma referência diferente. Kumar et al. (2006) sugerem a integração do Seis Sigma com o conceito de produção enxuta. Chang (2002) propõe um processo que parte do planejamento estratégico e o retroalimenta com os resultados dos projetos Seis Sigma. O modelo de implantação da Process Quality Associates (2003) baseia-se no próprio método de melhoria do Seis Sigma com suas fases DMAIC (define, measure, analyse, improve, contro).

\subsection{Fatores Críticos de Sucesso do Seis Sigma em PME}

Vários autores estudaram os fatores críticos de sucesso que uma PME deve atender na implantação do Seis Sigma. Entretanto, eles mesmos reconhecem que boa parte destes fatores são os mesmos observados nas grandes empresas tais como: comprometimento gerencial, alinhamento com as necessidades dos clientes, critério para seleção e priorização de projetos, reconhecimento dos resultados, treinamento adequado, mudança cultural (SPANYI; WURTZEL, 2003; DAVIES, 2005; PYZDEK, 2005; PARODY; VOELKEL, 2006).

Considerando as restrições financeiras da PME e a necessidade de retorno no curto prazo, Parody e Voelkel (2006) indicam que o processo de seleção e implantação de projetos Seis Sigma deve priorizar aqueles de alto impacto no resultado, mas com um tempo não muito longo para a sua realização. Em sentido contrário a estas restrições, Pyzdek (2005) entende que, para a PME implantar o Seis Sigma, ela precisa dispor de recursos excedentes. 


\subsection{Adaptações do Seis Sigma para Implantação em PME}

A maior parte das adaptações recomendadas pelos autores gira em torno do treinamento e da alocação dos especialistas. Com o avanço da tecnologia, Harry e Crawford (2004) indicam o treinamento online para as PME que irão implantar o Seis Sigma, pois este tipo de treinamento pode ser financeiramente viável para um número pequeno de pessoas. Os mesmos autores e Wessel e Burcher (2004) também sugerem a utilização de um treinamento por módulos, que permite fazer a customização do treinamento de acordo com as necessidades e capacidades da empresa. Godeiro et al. (2005) recomendam a simplificação do treinamento atendendo às restrições orçamentárias das PME.

Juntamente com o treinamento, a alocações dos especialistas encarregados do Seis Sigma é o fator que mais exige adaptações para implantação do programa em uma PME. Uma adaptação recomendada é a utilização de uma nova categoria de especialistas chamada white belts, que requer bem menos treinamento e oferece retorno mais rápido do que a formação de especialistas mais graduados (HARRY; CRAWFORD, 2004). Outra opção das PME é, ao contrário do que é defendido em livros-textos, a utilização dos black belts (os especialistas mais graduados) em regime de tempo parcial (NONTHALEERAK; HENDRY, 2008).

Wessel e Burcher (2004) e Esain et al. (2005) concluem em seus trabalhos que todo o programa deve focar no retorno de curto prazo. Para isto, segundo os mesmos autores, o esforço de acompanhamento dos resultados deve ser reduzido a no máximo 12 meses e o critério de seleção de projetos deve ser alinhado com a estratégia de retorno de curto prazo.

\subsection{Comparação da Implantação do Seis Sigma em PME e Grandes Empresas}

Uma das principais diferenças entre pequenas e grandes empresas, segundo De Feo (2002), é a forma de treinamento dos funcionários nas ferramentas e técnicas do Seis Sigma, considerando-se as restrições de recursos da PME. Além disso, o tempo necessário para empreender um projeto em uma PME poderá ser maior do que em uma grande empresa, dado que a PME não pode dispor de muitos funcionários em um pequeno período de tempo para implementar as melhorias. Em uma PME, o proprietário-dirigente deve ser o líder do programa Seis Sigma. Em uma grande empresa, esta função pode ser delegada a um executivo sênior. As principais vantagens das PME em relação às grandes empresas na adoção do programa Seis Sigma podem ser resumidas em: maior proximidade com os clientes, menor número de frentes de implantação, menor número de níveis hierárquicos, comunicação interna mais rápida e efetiva, forte influência do proprietário (WILSON, 2004).

\subsection{Estudos de Casos da Adoção do Seis Sigma em PME}

Estudos de caso da adoção do Seis Sigma em PME foram realizados nos mais diversos tipos de empresa e setores, tais como fazenda produtora de leite (TYLUTKI; FOX, 2002), indústria de equipamentos de teste para manutenção de aviões (GUPTA; SCHULTZ, 2005), empresa do ramo alimentício (SAMPAIO et al., 2005), cluster de oito empresas do setor automotivo (PANTANO et al., 2006), PME da Malásia do seguimento de alimento e bebida (SEOW, 2006), indústria inglesa de mobiliários para escritório (THOMAS; BARTON, 2006). Isto mostra que, além do porte da empresa, o Seis Sigma não se restringe a setores de atividade nem a localizações geográficas.

O estudo de vários casos de implantação do Seis Sigma em PME revela que este programa pode propiciar bons resultados, sejam estes financeiros ou de qualidade, ajudando a superar dificuldades dessas empresas. Contudo, tais resultados não vêm sem que os dirigentes das PME enfrentem desafios, dentre os quais: a implantação lenta devido à limitação de recursos e falta de dados precisos para análise (TYLUTKl; FOX, 2002); precariedade quanto à cultura para a qualidade; baixa qualificação dos funcionários; ausência de dados históricos, de controles sobre os processos e de recursos financeiros para investir no programa (SAMPAIO et al., 2005).

Sampaio et al. (2005) lembram que o custo de implantação do Seis Sigma em PME no Brasil pode ser amenizado com a ajuda do Sebrae ou de universidades.

\subsection{Situação Atual e Resultados Obtidos por PME que Adotaram o Seis Sigma}


Apesar de vários estudos confirmarem a aplicabilidade do Seis Sigma em PME, como Antony (2008), em levantamento aplicado em uma amostra de PME inglesas, Antony et al. (2005) identificaram que os dirigentes de grande quantidade dessas empresas ( $37 \%$ da amostra) não conheciam o Seis Sigma ou alegavam não possuir recurso para a sua implantação (26\%). Estes dados, pelo menos em relação à Inglaterra, indicam uma penetração ainda muito baixa do Seis Sigma em PME. Contudo, a maior parte dos dirigentes de PME que já adotou o Seis Sigma reporta ganhos financeiros (ANTONY, 2005; ADEYEMI, 2005) até mesmo consideráveis quando comparados proporcionalmente aos obtidos nas grandes empresas (KUMAR, 2007) e, em certos casos, com resultados mais rápidos e visíveis (ANTONY, 2008). Vale destacar, entretanto, que, na maioria dos casos, os ganhos na área de qualidade ainda são inferiores aos obtidos em grandes empresas (WILLIAMS, 2006).

Além de ganhos financeiros, Adeyemi (2005) identificou ganhos na satisfação de colaboradores e clientes na maioria das PME pesquisadas.

Com relação a aspectos importantes para se obter bons resultados, relatados em PME que já adotaram - Seis Sigma, identificou-se o apoio da alta administração (ADEYEMI, 2005; ANTONY et al., 2005; KUMAR, 2007), a realização de treinamentos “dentro de casa" por outros especialistas (ADEYEMI, 2005), alinhamento do programa com as necessidades dos clientes e com o planejamento estratégico (ANTONY et al., 2005).

\subsection{Seis Sigma como Estratégia de Negócios nas PME}

Camgoz-Akdag (2007) apresenta resultados de um survey aplicado em pequenas indústrias de eletroeletrônicos da Turquia. A questão final do estudo buscava identificar qual a melhor estratégia para uma PME local tornar-se uma indústria de classe mundial. A resposta encontrada pelo autor foi o "six sigma benchmarking". Segundo o autor, este conceito implica na aplicação de melhoria contínua através do Seis Sigma em conjunto com a utilização das melhores práticas do mercado através do "benchmarking".

\section{Classificação da Literatura sobre Seis Sigma em PME}

\subsection{Modelo de Classificação da Literatura}

Nesta seção, o modelo utilizado para classificar os 41 artigos revisados sobre Seis Sigma em PME é descrito. As categorias a seguir foram utilizadas para descrever sucintamente cada artigo analisado segundo o tema central do qual ele trata:

- MO - Modelos: trabalhos que sugerem modelos, frameworks ou métodos detalhados com o passo a passo para se implantar o programa Seis Sigma nas PME;

- FCS - Fatores Críticos: trabalhos que descrevem fatores críticos de sucesso para se implantar eficazmente o Seis Sigma nas PME;

- AD - Adaptações: trabalhos que analisam as principais adaptações necessárias para implantação do Seis Sigma no contexto de uma PME;

- CO - Comparação: trabalhos que compararam as principais características entre a adoção do Seis Sigma nas PME e nas grandes empresas;

- CA - Casos: trabalhos que descrevem casos de utilização do Seis Sigma em PME;

- RE - Resultados: trabalhos que avaliam a situação atual de PME que adotaram o Seis Sigma e os principais resultados obtidos com esse programa;

- ES - Estratégia: trabalhos que avaliam a opção das empresas adotarem o Seis Sigma como uma estratégia de negócios;

- TE - Teoria: trabalhos que objetivaram criar uma teoria que explica o fenômeno Seis Sigma nas PME.

Cada trabalho identificado como pertinente foi também classificado segundo seu ano de publicação, sua origem de publicação (nacional ou internacional) e seu tipo de trabalho, de acordo com os seguintes itens: 
- AA - Artigo acadêmico: trabalho publicado em revista acadêmica, na qual informações obtidas de outras fontes são citadas no texto e detalhadas nas referências bibliográficas;

- AN - Artigo não acadêmico: trabalhos produzidos para revistas especializadas ou para comunidades ou fóruns de usuários nos quais as fontes das informações não são citadas no texto;

- CA - Congresso acadêmico: artigos com rigor acadêmico publicados em anais de congressos;

- TD - Teses/Dissertações: teses de doutorado ou dissertações de mestrado.

Os trabalhos também foram classificados quanto ao método de pesquisa adotado. A explicação abaixo de cada método é adaptada de Nonthaleerak e Hendry (2006):

- DE - Descritivo: trabalho que explica ou descreve vários aspectos do Seis Sigma sem o embasamento de informações de pesquisa empírica de campo - como casos, por exemplo;

- EM - Empírico: trabalho baseado em dados obtidos em campo; pode ser um estudo de caso, experimental, exploratório, longitudinal ou baseado em um levantamento (survey); o trabalho baseia-se em um método estruturado utilizando técnicas de pesquisa tais como entrevistas ou questionários;

- CO - Conceitual: $t$ rabalho com o propósito de explicar, propor ou desenvolver um novo modelo conceitual sem o teste deste com dados empíricos;

- RL - Revisão da literatura: trabalho que foca na revisão da literatura.

\subsection{Síntese da Classificação da Literatura sobre Seis Sigma em PME}

A seguir é apresentada uma síntese dos 41 artigos revisados agrupados segundo o tema central de que tratam, ou seja, MO, FC, AD, CO, CA, RE, ES ou TE. Para cada um deles é apresentado, na tabela, o ano de publicação, o tipo do trabalho, o método de pesquisa, a sua origem (nacional ou internacional) e uma breve síntese da maior contribuição do estudo.

Dos 41 trabalhos revisados, nove focaram no tema "modelos para implantação do Seis Sigma no contexto de uma PME". Estes trabalhos não só reforçam a aplicabilidade do Seis Sigma ao contexto das PME, como também propõem práticas para que esta implantação seja bem sucedida. A tabela 1 sintetiza estes trabalhos.

Tabela 1 - Trabalhos sobre "Modelos de implantação do Seis Sigma em PME"

\begin{tabular}{|c|c|c|c|c|c|c|}
\hline Autores & Ano & Tema & Tipo & Método & Origem & Contribuições \\
\hline GROSS & 2001 & MO & AN & DE & IN & $\begin{array}{l}\text { Road map proposto para grandes empresas mas } \\
\text { que as PME também podem seguir }\end{array}$ \\
\hline CHANG & 2002 & MO & TD & EM & IN & $\begin{array}{l}\text { Framework de implantação que parte do } \\
\text { planejamento estratégico e o retroalimenta }\end{array}$ \\
\hline DAVIS & 2003 & MO & AN & DE & IN & $\begin{array}{l}\text { Forma bem resumida de } 6 \text { passos para implantar } \\
\text { o seis sigma em PME }\end{array}$ \\
\hline $\begin{array}{l}\text { PROCESS QUALITY } \\
\text { ASSOCIATES }\end{array}$ & 2003 & MO & AN & DE & IN & $\begin{array}{l}\text { Framework para PME que considera suas } \\
\text { características positivas e negativas }\end{array}$ \\
\hline SCHWINN & 2003 & MO & AN & DE & IN & Six Sigma Simplified (S3) customizado para PME. \\
\hline BURTON & 2004 & MO & AN & DE & IN & $\begin{array}{l}\text { Modelo escalonado de implementação com pouco } \\
\text { investimento e sem estrutura adicional }\end{array}$ \\
\hline PINHO & 2005 & MO & TD & EM & NA & $\begin{array}{l}\text { Modelo de aplicação do programa seis sigma em } \\
\text { PME dividido em três fases }\end{array}$ \\
\hline KUMAR et al. & 2006 & MO & AA & EM & IN & $\begin{array}{l}\text { Propõe um framework Lean Sigma(integra os } \\
\text { conceitos da produção enxuta com seis sigma) }\end{array}$ \\
\hline THOMAS & 2006 & MO & AN & DE & IN & $\begin{array}{l}\text { Roteiro que os CEOs devem seguir para } \\
\text { implementar o seis sigma em uma PME }\end{array}$ \\
\hline
\end{tabular}

Dos 41 trabalhos revisados, seis focaram na descrição dos fatores críticos de sucesso para implantação do Seis Sigma em uma PME. A tabela a seguir sintetiza estes trabalhos. 
Tabela 2 - Trabalhos sobre o tema "Fatores críticos de sucesso do Seis Sigma em PME"

\begin{tabular}{|c|c|c|c|c|c|c|}
\hline Autores & Ano & Tema & Tipo & Método & Origem & Contribuições \\
\hline KELLER & 2003 & FC & AN & DE & IN & $\begin{array}{l}\text { Importância da alocação de recursos e } \\
\text { Implantação escalonada pelo \% empregados }\end{array}$ \\
\hline SPANYI e WURTZEL & 2003 & FC & AN & DE & IN & $\begin{array}{l}\text { Utilização das ferramentas mais simples do seis } \\
\text { sigma e FCS (mesmos das grande empresas) }\end{array}$ \\
\hline DAVIES & 2005 & FC & AN & DE & IN & $\begin{array}{l}\text { Abordagem mais flexível para treinar e alocar os } \\
\text { black belts. }\end{array}$ \\
\hline PYZDEK & 2005 & FC & AN & DE & IN & $\begin{array}{l}\text { Alocação de } 0,5 \% \text { a } 1 \% \text { do total de horas no } \\
\text { programa. Recursos excedentes e redundância. }\end{array}$ \\
\hline $\begin{array}{l}\text { PARODY e } \\
\text { VOELKEL }\end{array}$ & 2006 & FC & AN & DE & IN & $\begin{array}{l}3 \text { ingredientes: comprometimento gerencial; } \\
\text { projetos curtos de alto impacto e líder experiente }\end{array}$ \\
\hline MAIRANI & 2007 & FC & AN & DE & IN & $\begin{array}{l}\text { Um sistema de gestão da qualidade previamente } \\
\text { instalado e funcionando. }\end{array}$ \\
\hline
\end{tabular}

Foram identificados seis trabalhos que tratam das adaptações necessárias para usar o Seis Sigma nas PME, como se vê na tabela 3, abaixo.

Tabela 3 - Trabalhos sobre tema "Adaptações do método Seis Sigma para a PME"

\begin{tabular}{|c|c|c|c|c|c|c|}
\hline Autores & Ano & Tema & Tipo & Método & Origem & Contribuições \\
\hline $\begin{array}{l}\text { HARRY e } \\
\text { CRAWFORD }\end{array}$ & 2004 & $A D$ & AN & DE & IN & $\begin{array}{l}\text { Treinamento online e modularizado. Nova } \\
\text { categoria de especialistas: white belt }\end{array}$ \\
\hline ROWLANDS et al. & 2004 & $A D$ & CA & DE & IN & $\begin{array}{l}\text { Utilização do black belt deve levar em } \\
\text { consideração a disponibilidade de recursos. }\end{array}$ \\
\hline $\begin{array}{l}\text { WESSEL e } \\
\text { BURCHER }\end{array}$ & 2004 & $A D$ & AA & EM & IN & $\begin{array}{l}10 \text { requerimentos funcionais para adaptação do } \\
\text { programa seis sigma para } \mathrm{PME}\end{array}$ \\
\hline ESAIN et al. & 2005 & $A D$ & CA & EM & IN & $\begin{array}{l}\text { Mudanças na abordagem utilizada para treinar } \\
\text { black belt's em PME }\end{array}$ \\
\hline GODEIRO et al. & 2005 & $A D$ & CA & RL & NA & $\begin{array}{l}\text { Treinamento reduzido, terceirização de Black } \\
\text { Belts, ferramentas mais simples e software livre }\end{array}$ \\
\hline $\begin{array}{l}\text { NONTHALEERAK e } \\
\text { HENDRY }\end{array}$ & 2008 & $A D$ & AA & EM & IN & $\begin{array}{l}\text { Utilização do black belt em regime parcial de } \\
\text { tempo }\end{array}$ \\
\hline
\end{tabular}

Apenas os três trabalhos resumidos na tabela 4 avaliam explicitamente as diferenças de implantação do Seis Sigma em PME e em grandes empresas.

Tabela 4 - Trabalhos sobre o tema "Comparação do Seis Sigma em PME e grandes empresas"

\begin{tabular}{lllllll}
\hline Autores & Ano & Tema & Tipo & Método & Origem & Contribuições \\
\hline WAXER & 2001 & CO & AN & DE & IN & $\begin{array}{l}\text { Comprometimento gerencial e reconhecimento é } \\
\text { mais fácil; treinamento e alocação dos } \\
\text { especialistas é mais difícil em PME }\end{array}$ \\
DE FEO & 2002 & CO & AN & DE & IN & $\begin{array}{l}\text { Magnitude menor dos projetos; maior tempo de } \\
\text { projeto; proprietário deve ser o champion }\end{array}$ \\
WILSON & 2004 & CO & AN & DE & IN $\begin{array}{l}\text { Cliente e proprietário próximos, menor número de } \\
\text { sites, menos níveis hierárquicos, melhor } \\
\text { comunicação interna }\end{array}$ \\
\hline
\end{tabular}

Dentre os 41 trabalhos revisados, identificamos nove estudos de caso em PME que adotaram o Seis Sigma, como indica a síntese da tabela 5 . 
Tabela 5 - Trabalhos sobre o Tema "Casos de Adoção do Seis Sigma em PME"

\begin{tabular}{|c|c|c|c|c|c|c|}
\hline Autores & Ano & Tema & Tipo & Método & Origem & Contribuições \\
\hline TYLUTKI e FOX & 2002 & CA & AN & DE & IN & $\begin{array}{l}\text { Implantação lenta devido a limitação de recursos e } \\
\text { falta de dados acurados para análise }\end{array}$ \\
\hline GNIBUS e KRULL & 2003 & CA & AN & DE & IN & $\begin{array}{l}\text { Inicialmente basta treinar green belts; é uma } \\
\text { ferramenta disciplinada para melhorar resultados }\end{array}$ \\
\hline GUPTA e SCHULTZ & 2005 & CA & AN & $\mathrm{DE}$ & IN & $\begin{array}{l}\text { Empresa aferiu ganhos significativos: financeiros } \\
\text { e de qualidade }\end{array}$ \\
\hline SAMPAIO et al. & 2005 & CA & CA & EM & NA & $\begin{array}{l}\text { Metodologia é eficaz para melhorias. Dificuldades: } \\
\text { cultura, falta qualificação e dados }\end{array}$ \\
\hline GREEN et al. & 2006 & CA & AA & EM & IN & $\begin{array}{l}\text { Treinamento para um nível menor que o green } \\
\text { belt }\end{array}$ \\
\hline PANTANO et al. & 2006 & CA & CA & EM & IN & $\begin{array}{l}\text { Ênfase em elementos da essência do seis sigma } \\
\text { em oposição a ferramentas sofisticadas }\end{array}$ \\
\hline SEOW & 2006 & CA & CA & EM & IN & $\begin{array}{l}\text { Customização do programa seis sigma para a } \\
\text { realidade de uma PME da Malásia }\end{array}$ \\
\hline THOMAS E BARTON & 2006 & CA & AA & EM & IN & $\begin{array}{l}\text { Empresa estudada superou os desafios } \\
\text { normalmente impostos a PME }\end{array}$ \\
\hline THOMAS E LEWIS & 2007 & CA & AA & EM & IN & $\begin{array}{l}\text { Aplica um estratégia combinada de TPM e seis } \\
\text { sigma em uma PME }\end{array}$ \\
\hline
\end{tabular}

Sete trabalhos analisados focaram na avaliação da situação atual e dos resultados obtidos pelas PME que implantaram o Seis Sigma - como exposto na tabela 6.

Tabela 6 - Trabalhos sobre "Situação Atual e Resultados Obtidos por PME que Adotaram o Seis Sigma"

\begin{tabular}{|c|c|c|c|c|c|c|}
\hline Autores & Ano & Tema & Tipo & Método & Origem & Contribuições \\
\hline ADEYEMI & 2005 & RE & TD & EM & IN & $\begin{array}{l}\text { Melhor satisfação de colaboradores e clientes, } \\
\text { aumento da rentabilidade; Treinamento interno }\end{array}$ \\
\hline ANTONY et al. & 2005 & RE & AA & EM & IN & $\begin{array}{l}\text { Muitas PME não conhecem o seis sigma; ou não } \\
\text { possuem recursos para implementar }\end{array}$ \\
\hline WILLIAMS & 2006 & RE & AN & EM & IN & $\begin{array}{l}\text { Resultados financeiros superiores a grandes } \\
\text { empresas mas qualidade inferior }\end{array}$ \\
\hline KUMAR & 2007 & RE & AA & EM & IN & $\begin{array}{l}\text { Comprometimento gerencial é FCS, treinamento } \\
\text { fraco e disponibilidade de recursos são } \\
\text { obstáculos, obtém benefícios como as grandes }\end{array}$ \\
\hline MARX & 2007 & RE & AN & EM & IN & $\begin{array}{l}\text { Projetos mais rápidos; especialistas mais } \\
\text { independentes; }\end{array}$ \\
\hline ANTONY & 2008 & RE & AA & EM & IN & $\begin{array}{l}\text { Seis Sigma é aplicável; Resultados são mais } \\
\text { rápidos e mais visíveis }\end{array}$ \\
\hline ULMER & 2008 & RE & TD & EM & IN & $\begin{array}{l}\text { Identificação de correlação entre tempo de } \\
\text { escolaridade e melhoria dos custos da qualidade }\end{array}$ \\
\hline
\end{tabular}

Pudemos identificar apenas um trabalho que trata do Seis Sigma como uma estratégia de negócios.

Tabela 7 - Trabalho sobre "Adoção do Seis Sigma como uma estratégia de negócios"

\begin{tabular}{lcccccc}
\hline Autores & Ano & Tema & Tipo & Método & Origem & Contribuições \\
\hline CAMGOZ-AKDAG & 2007 & ES & AA & EM & IN & $\begin{array}{l}\text { Identificar a melhor estratégia para uma PME } \\
\text { tornar-se uma indústria de classe mundial }\end{array}$ \\
\hline
\end{tabular}

Nenhum trabalho foi encontrado nas bases analisadas com o foco no desenvolvimento de uma fundamentação teórica para tratar com profundidade do uso do Seis Sigma especificamente no contexto das PME.

\section{Análise das Pesquisas sobre Seis Sigma em PME e Recomendações para Novas Pesquisas}

Nesta seção, é realizada uma análise dos 41 trabalhos publicados sobre o tema Seis Sigma em PME, considerando as dimensões ano de publicação, origem do trabalho (nacional/internacional), o método utilizado no trabalho (empírico, descritivo, revisão da literatura ou conceitual), o tema enfocado e o tipo do 
trabalho (artigo acadêmico, artigo não acadêmico, publicação de congresso ou tese/dissertação). Além disso, são identificadas oportunidades para o desenvolvimento de futuras pesquisas.

A figura 2 apresenta a quantidade de trabalhos produzidos por ano e a discriminação das publicações indicando se são nacionais ou internacionais.

Em artigo que contemplou uma revisão da literatura do Seis Sigma como um todo, não especificamente nas PME, Nonthaleerak e Hendry (2006) identificaram os primeiros trabalhos sobre esse tema a partir de 1991. Observa-se no artigo destes autores que a publicação sobre o tema associada às PME iniciou-se apenas 10 anos depois, ou seja, em 2001, e atingiu seu ápice nos anos de 2005 e 2006 com nove e oito produções respectivamente. Destaca-se também que a grande maioria dos artigos (38) foram produzidos fora do Brasil. Foram encontrados apenas três artigos nacionais, sendo todos em 2005.

Figura 2 Quantidade de artigos nacionais e internacionais publicados por ano

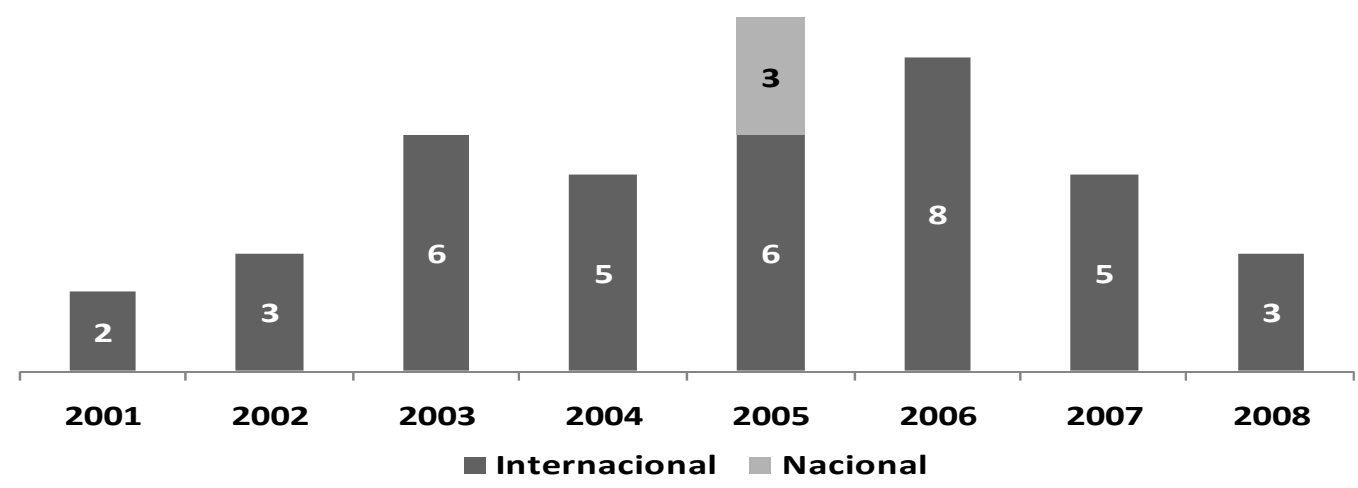

Identifica-se facilmente que a produção brasileira sobre o tema é muito restrita, o que sugere uma necessidade de pesquisa sobre o Seis Sigma em PME nacionais.

Na figura 3 a seguir, verificamos a quantidade de artigos acadêmicos e não acadêmicos produzidos ano a ano. Foram considerados como trabalhos acadêmicos artigos feitos em contexto acadêmico, publicações de congressos acadêmicos e teses/dissertações. Os demais tipos de trabalho foram vistos como não acadêmicos.

Figura 3 - Quantidade de artigos acadêmicos e não acadêmicos publicados por ano

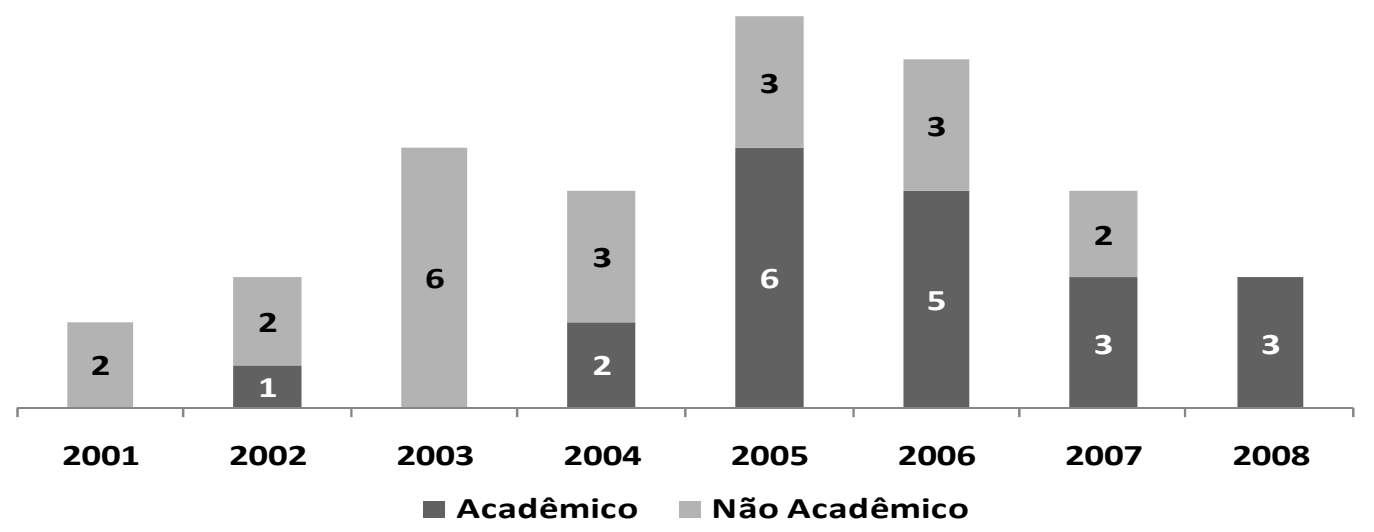

Podemos destacar, com a análise desta figura, que os trabalhos não acadêmicos foram maioria entre os anos de 2001 e 2004 e a situação se inverteu entre os anos 2005 e 2008. Uma das possibilidades para explicar este fenômeno é o tempo maior necessário para os pesquisadores acadêmicos realizarem a 
pesquisa de campo (como veremos a frente, a grande maioria destas pesquisas se basearam em trabalhos empíricos) e pelo fato dos artigos acadêmicos demandarem mais tempo para serem publicados, dada a necessidade de serem avaliados por pares em meio acadêmico e frequentemente deverem ser aperfeiçoados após uma primeira avaliação. Outra hipótese explicativa é que o aumento da produção técnica tenha suscitado interesse posterior nos acadêmicos, que, a partir de então, podem ter iniciado suas publicações sobre o tema.

A tabela 8 detalha os dados relatados acima, porém estratificando os trabalhos acadêmicos em artigos, publicações de congresso e teses/dissertações. Verificamos que a maioria dos trabalhos acadêmicos que identificamos são artigos publicados em revistas acadêmicas (24\%), contra seis artigos publicados em anais de congressos (15\%) e apenas quatro testes/dissertações (10\%). Mas percebe-se um equilíbrio entre o total de trabalhos acadêmicos (artigos de revistas + artigos de congresso + teses / dissertações) e não acadêmicos com 20 (49\%) e 21 (51\%) trabalhos respectivamente.

Tabela 8 - Quantidade de artigos por tipo publicados por ano

\begin{tabular}{|lcccccccc|c|c|}
\hline Tipo & 2001 & 2002 & 2003 & 2004 & 2005 & 2006 & 2007 & 2008 & Total & $\%$ \\
\hline Artigo Acadêmico & & & & 1 & 1 & 3 & 3 & 2 & 10 & $24 \%$ \\
Congresso Acadêmico & & & & 1 & 3 & 2 & & & 6 & $15 \%$ \\
Teses e Dissertações & & 1 & & & 2 & & & 1 & 4 & $10 \%$ \\
Artigo Não Acadêmico & 2 & 2 & 6 & 3 & 3 & 3 & 2 & & 21 & $51 \%$ \\
\hline Total & 2 & 3 & 6 & 5 & 9 & 8 & 5 & 3 & 41 & $100 \%$ \\
\hline
\end{tabular}

A tabela 9, a seguir, apresenta a quantidade de artigos cruzando o tipo (artigo acadêmico, artigo de congresso, teses/dissertações e artigos não acadêmicos) com o método utilizado nos estudos. Como era de se esperar, a grande maioria dos artigos não acadêmicos são meramente descritivos ou normativos, não se baseando em pesquisas empíricas (19 de 21 trabalhos). Por outro lado, conforme já adiantado acima, os trabalhos acadêmicos utilizaram em sua maioria uma pesquisa empírica (estudos de caso e survey principalmente), totalizando 18 de 20 trabalhos. É importante destacar que não foi encontrado qualquer trabalho de foco conceitual. Desta forma, esta coluna não foi incluída na tabela 9.

Tabela 9 - Quantidade de artigos por tipo e por método de pesquisa

\begin{tabular}{|lccc|c|}
\hline Tipo & Descritivo & Empírico & $\begin{array}{c}\text { Revisão } \\
\text { Literatura }\end{array}$ & Total \\
\hline Artigo Acadêmico & & 10 & & 10 \\
Congresso Acadêmico & 1 & 4 & 1 & 6 \\
Teses/Dissertações & & 4 & & 4 \\
Artigo Não Acadêmico & 19 & 2 & & 21 \\
\hline Total & 20 & 20 & 1 & 41 \\
$\%$ & $49 \%$ & $49 \%$ & $2 \%$ & $100 \%$ \\
\hline
\end{tabular}

A figura 4 apresenta um gráfico com a quantidade de trabalhos por tema de estudo, dividindo cada barra com a quantidade por método de pesquisa (descritivo, empírico, e revisão da literatura). Nela, percebe-se que os temas mais pesquisados foram "modelos para implantação do Seis Sigma em PME" e "estudos de casos de implantação do Seis Sigma em PME" - com nove trabalhos para cada um destes temas. Por outro lado, observa-se que os temas "utilização do Seis Sigma como estratégia competitiva em PME" e "comparação do Seis Sigma entre pequenas e grandes empresas" foram os menos pesquisados, com um e três trabalhos respectivamente. Aí reside outra oportunidade para futuras pesquisas que podem explorar melhor a questão de como o Seis Sigma pode ser adotado para auxiliar a estratégia de negócios em PME. 
Figura 4 - Quantidade de trabalhos por tema e por método de pesquisa

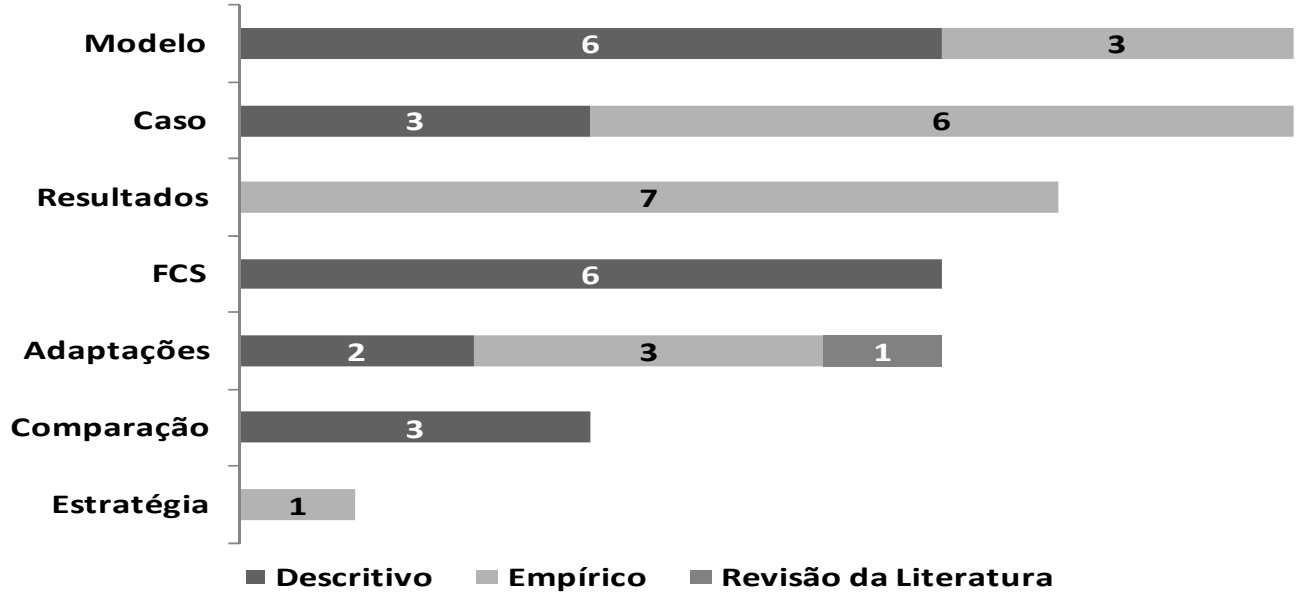

Outro ponto a destacar em relação à figura 4 é que não foi possível identificar nem ao menos uma pesquisa empírica para os temas "fatores críticos de sucesso do Seis Sigma em PME" e "comparação do Seis Sigma entre pequenas e grandes empresas". Estes temas são, portanto, o centro de grandes necessidades/oportunidades de pesquisa.

A tabela 10 apresenta a quantidade de trabalhos acadêmicos e não acadêmicos segundo o tema dos trabalhos. Analisando os dados, pode-se confirmar as oportunidades já destacadas para pesquisas acadêmicas a respeito dos temas "fatores críticos de sucesso" e "comparação PME e grandes empresas". Podemos também identificar a oportunidade de desenvolvimento de mais trabalhos acadêmicos sobre o tema "modelo para implantação de Seis Sigma em PME", pois, dos nove trabalhos publicados a esse respeito, apenas três foram acadêmicos.

Tabela 10 - Quantidade de trabalhos por tema e por tipo de trabalho

\begin{tabular}{|lcc|cc|}
\hline Tema & Acadêmico & $\begin{array}{c}\text { Não } \\
\text { Acadêmico }\end{array}$ & Total & $\%$ \\
\hline Modelo & 3 & 6 & 9 & $22 \%$ \\
Casos & 6 & 3 & 9 & $22 \%$ \\
Resultados & 5 & 2 & 7 & $17 \%$ \\
FCS & & 6 & 6 & $15 \%$ \\
Adaptações & 5 & 1 & 6 & $15 \%$ \\
Comparação & & 3 & 3 & $7 \%$ \\
Estratégia & 1 & & 1 & $2 \%$ \\
Teoria & & & 0 & $0 \%$ \\
\hline Total & 20 & 21 & 41 & $100 \%$ \\
\hline
\end{tabular}

Os dados mostram ainda a necessidade de realização de trabalhos acadêmicos que possam desenvolver abordagens conceituais aprofundadas acerca da realização de programas Seis Sigma no contexto das PME, levando-se em consideração as particularidades destas organizações - não identificamos trabalhos já realizados com este foco, mesmo que o tema seja de grande importância.

\section{Conclusões}

O programa Seis Sigma pode trazer contribuições para as empresas que o adotam. Apesar de ele ter sido projetado inicialmente para grandes empresas e os relatos dos ganhos obtidos focarem este tipo de empresa, podemos verificar, através dos resultados das pesquisas revisadas neste trabalho, que estes benefícios também podem ser obtidos pelas PME. Contudo, a busca destes benefícios poderá ser facilitada 
pela produção de mais conhecimentos acadêmicos aprofundados sobre o tema e sua utilização nestas organizações, que guardam numerosas diferenças em relação às grandes empresas.

Atingindo nossos dois objetivos propostos neste artigo, pudemos avaliar e descrever o "estado da arte" dos estudos sobre o Seis Sigma em PME, assim como identificar lacunas a serem exploradas em futuras pesquisas acadêmicas sobre o tema, tais como:

- Pesquisas sobre a realidade do Seis Sigma em PME no Brasil;

- Avaliação de como o Seis Sigma pode ser adotado como apoio à estratégia de negócios em PME;

- Trabalhos acadêmicos com pesquisa empírica para se confirmar os fatores críticos de sucesso de implantação do Seis Sigma nas PME e também para se identificar novos fatores críticos;

- Pesquisa empírica para entender e identificar as principais diferenças entre a implantação do Seis Sigma nas PME e nas grandes empresas;

- Trabalhos conceituais ou teóricos aprofundados tratando das particularidades das PME e suas influências sobre o uso do Seis Sigma nestas organizações.

Esperamos que esta revisão da literatura possa facilitar e estimular novos pesquisadores a buscar a evolução do entendimento de como o programa Seis Sigma pode ser útil para os pequenos e médios empreendimentos.

\section{Referências}

ADEYEMI, Y. An analysis of six sigma at small vs. large manufacturing companies. 2005. $79 \mathrm{f}$. Thesis (Master in Industrial Engineering) - University of Pittsburgh, Pittsburgh, 2005.

ANTONY, J. Can Six Sigma be effectively implemented in SMEs? International Journal of Productivity and Performance Management, v. 57, n. 5, p. 420-423, 2008.

ANTONY, J.; KUMAR, M.; MADU, C. N. Six sigma in small- and medium-sized UK manufacturing enterprises: Some empirical observations. The International Journal of Quality \& Reliability Management, v. 22, n. 8-9, 2005.

BAÑUELAS, R.; ANTONY, J. Critical success factors for the successful implementation of six sigma projects in organizations. The TQM Magazine, v. 14, n. 2, p. 92-99, 2002.

BARNEY, M.; MC CARTY, T. The new six sigma: a leader's guide to achieving rapid business improvement and sustainable results. New Jersey: Prentice Hall, 2002.

BASU, R.; WRIGHT J. N. Quality beyond six sigma. Oxford: Butterworth-Heinemann, 2003.

BURTON, T. T. Six sigma for small and medium sized businesses. 2004. Disponível em <http://www.isixsigma.com/library/content/c030224a.asp>. Acesso em 02 maio 2008.

CAMGOZ-AOKDAG, H. Total quality management through six sigma benchmarking: A case study. Benchmarking, v. 14, n. 2, p. 186-201, 2007.

CHANG, T. Six Sigma: a framework for small and medium-sized enterprises to achieve total quality. 2002. 208 f. Thesis (PhD in Production Management). Cleveland State University, Cleveland, 2002.

DAVIES, E. C. South west region: six sigma for SMEs. Management Services, v. 49, n. 2, p. 6, 2005.

DE FEO, J. A. Ask the expert - the topic: six sigma deployment at large and small companies. 2002.

Disponível em: <http://www.isixsigma.com/library/content/ a020923a.asp> Acesso em 09/ jul. 2008.

DEMING, W. E. Out of the crisis. Cambridge: MIT Press, 2000.

DOW, D.; SAMSON, D.; FORD, S. Exploding the myth: do all quality management practices contribute to superior quality performance? Production and Operations Management, v. 8, n. 1, p. 1-27, 1999.

ESAIN, A.; CATHERWOOD, P.; ROWLANDS, H. A new approach to six sigma training - Welsh SME's needs and wants discussed. In: EUROMA INTERNATIONAL CONFERENCE ON OPERATIONS AND GLOBAL

COMPETITIVENESS. 2005, Budapest, Hungary. Proceedings... Brussels, Belgium, EurOMA, 2005. 
GODEIRO, D. P. O. et al. Cultura Seis Sigma em pequenas e médias empresas. In: ENCONTRO NACIONAL DE ENGENHARIA DE PRODUÇÃO, 26., 2006, Fortaleza. Anais... Rio de Janeiro, ABEPRO, 2006.

GODINHO FILHO, M.; FERNANDES, F. C. F. Manufatura enxuta: uma revisão que classifica e analisa os trabalhos apontando perspectivas de pesquisas futuras. Gestão \& Produção, v. 11, n. 1, p. 1-19, 2004.

GROSS, J. M. A road map to six sigma quality, Quality Progress, v. 34, n. 11, p. 24-30, 2001.

GUPTA, P.; SCHULTZ, B. Six Sigma successes in small business: the popular quality methodology brings improvement to ideal Aerosmith. Quality Digest, Abr, 2005. Disponível em:

<http://www.qualitydigest.com/april05/articles/02_article.shtml>. Acesso em: 18 abr. 2008.

HARRY, M. J.; CRAWFORD, J. D. Six sigma for the little guy. Mechanical Engineering. v. 126, n. 11, p. E8, nov., 2004.

JURAN, J. M. Juran on leadership for quality: an executive handbook. New York: Free Press, 1989.

KUMAR, M. Critical success factors and hurdles to six sigma implementation: the case of a UK manufacturing SME. International Journal of Six Sigma and Competitive Advantage. v. 3, n. 4, p. 333-351, 2007.

KUMAR, M. et al. Implementing the lean sigma framework in an Indian SME: a case study. Production Planning \& Control. v. 17, n. 4, p. 407-423, jun, 2006.

MAIRANI, J. No matter the plant size, quality management systems measure up. Plant Engineering. v. $61, n$. 4, p. 25, 2007.

NONTHALEERAK, P.; HENDRY, L.C. Six Sigma: literature review and key future research areas. International Journal of Six Sigma and Competitive Advantage, v. 2, n. 2, p. 105-161, 2006.

NONTHALEERAK, P.; HENDRY, L.C. Exploring the six sigma phenomenon using multiple case study evidence, International Journal of Operations \& Production Management, v. 28, n. 3, p. 279-303, 2008.

PANTANO, V.; O'KANE, P.; SMITH, K. Cluster-based six sigma deployment in small and medium sized enterprises. In: Management of Innovation and Technology, 2006 IEEE International Conference. v. 2, p. 788-792, jun, 2006.

PARODY, R. J.; VOELKEL, J. G. Six sigma start-up at small companies. Quality Progress, v. 39, n. 5, p. 68-69, maio, 2006.

PINHO, C. T. A. Seis sigma: uma proposta para implementação da metodologia em pequenas e médias empresas. 2005. Dissertação (Mestrado em Engenharia de Produção). Universidade Federal do Rio Grande do Norte, Rio Grande do Norte, 2005.

PROCESS QUALITY ASSOCIATES. Six sigma for SMEs. 2003. Disponível em: <http://www.pqa.net/ProdServices/sixsigma/W06002007.html> Acesso em: 12 jul. 2008.

PYZDEK, T. A Roadmap for deploying six sigma in small businesses. 2005. Disponível em: <http://www.isixsigma.com/library/content/c051024a.asp> Acesso em: 09 jul. 2008.

SAMPAIO, L. L.; COSTA, I. C.; SERRA, C. M. V. Análise da utilização da metodologia seis sigma na elaboração de um projeto de melhoria para uma pequena empresa: um estudo de caso. In: ENCONTRO NACIONAL DE ENGENHARIA DE PRODUÇÃO, 25., 2005, Fortaleza. Anais... Rio de Janeiro, ABEPRO, 2005.

SCHWINN, D. R. Six sigma simplified for small organizations. 2003. Disponível em:

<http://www.qualityadvisor.com/library/six_sigma_simplified_for_small_businesses.php>. Acesso em: 09 maio 2008.

SENAPATI, S. R. Six sigma: myths and realities. International Journal of Quality \& Reliability Management, v. 21, n. 6, p. 683-690, 2004.

SEOW, C. J. L. Innovation in maintenance strategy through six sigma: insights of a malaysian SME In: Management of Innovation and Technology, 2006 IEEE International Conference. v. 2, p. 793-797, jun., 2006. 
SPANYI, A.; WURTZEL, M. Six Sigma for the rest of us: it's possible to tailor this popular methodology to fit your organization's size and finances. 2003. Disponível em <http://www.qualitydigest.com>. Acesso em: 08 maio 2008.

THOMAS, A.; BARTON, R. Developing an SME based six sigma strategy. Journal of Manufacturing Technology Management. v. 17, n. 4, p. 417-434, 2006.

THOMAS, A.; LEWIS, G. Developing an SME-based integrated TPM-Six Sigma strategy. International Journal of Six Sigma and Competitive Advantage, v. 3, n. 3, p. 228-247, 2007.

THOMAS, D. What small business CEOs must know to start six sigma. 2006. Disponível em: <http://www.isixsigma.com/library/content/c060116a.asp> Acesso em: 09 jul. 2008.

TYLUTKI, D. P.; FOX, D. G. Mooooving toward Six Sigma. Quality Progress, v. 35, n. 2, p. 34-42, 2002.

WELSH, J. A.; WHITE, J. F. A small business is not a little big business. Harvard Business Review, v. 59, n. 4, p. 18-27, 1981 .

WERKEMA, M. C. C. Report seis sigma. São Paulo: EPSE, 2002.

WESSEL, G.; BURCHER, P. Six Sigma for small and medium-sized enterprises. The TQM Magazine, v. $16, \mathrm{n}$. 4, p. 264-272, 2004.

WILLIAMS, K. Is six sigma beneficial for midsize companies? Strategic Finance, v. 88, n. 5, p. 23, nov., 2006. WILSON, N. The small company and six sigma: advantages of small business culture. 2004. Disponível em: <www.sixsigmaforum.com/protected/articles/>. Acesso em 09 jul. 2008.

WOMACK, J. P.; JONES, D. T.; ROOS, D. A máquina que mudou o mundo. 14. ed. Rio de Janeiro: Campus, 1992. 